Supporting Information

\title{
Reaction of $\mathrm{Cp}_{2}{ }_{2} \mathrm{Zr}$ (2,3-dimethylbutadiene) with Isonitriles and CO
}

Travis N. Valadez, Jack R. Norton, ${ }^{*}$ Michelle C. Neary, and Patrick J. Quinlivan

Department of Chemistry, Columbia University, New York, New York 10027, United

States

\section{Table of Contents:}

Table S1. Crystal, Intensity, Collection, and Refinement Data for 2, 3, and 4 S2

Table S2. Crystal, Intensity, Collection, and Refinement Data for 5, 6, and 7 S3

Table S3. Crystal, Intensity, Collection, and Refinement Data for $\mathbf{1}$ and 8 S4

$\begin{array}{lr}\text { Figure S1. Molecular Structure of } 7 & \text { S5 }\end{array}$

Figure S2. Molecular Structure of $8 \quad$ S5

Figure S3. Molecular Structure of $\mathbf{1}$

$\begin{array}{ll}\text { NMR Spectra } & \text { S7 }\end{array}$ 
Table S1. Crystal, Intensity, Collection, and Refinement Data for 2-4

\begin{tabular}{|c|c|c|c|}
\hline & 2 & $3 \cdot 0.5\left(\mathrm{C}_{7} \mathrm{H}_{8}\right)$ & 4 \\
\hline lattice & Orthorhombic & Triclinic & Monoclinic \\
\hline formula & $\mathrm{C}_{31} \mathrm{H}_{49} \mathrm{NZr}$ & $\mathrm{C}_{40.5} \mathrm{H}_{59} \mathrm{NZr}$ & $\mathrm{C}_{34} \mathrm{H}_{54} \mathrm{~N}_{2} \mathrm{Zr}$ \\
\hline formula weight & 526.93 & 651.10 & 582.01 \\
\hline space group & $P b c a$ & $P-1$ & $P 2_{1} / n$ \\
\hline$a / \AA ̊$ & $15.033(2)$ & $9.9539(4)$ & $11.5076(5)$ \\
\hline$b / \AA$ & $14.892(2)$ & $10.2631(4)$ & $15.1678(7)$ \\
\hline$c / \AA ̊$ & $25.247(4)$ & $17.0973(7)$ & $18.0604(8)$ \\
\hline$\alpha /^{\circ}$ & 90 & $93.2340(10)$ & 90 \\
\hline$\beta /^{\circ}$ & 90 & $100.7900(1)$ & $104.1020(10)$ \\
\hline$\gamma /^{\circ}$ & 90 & $97.6760(10)$ & 90 \\
\hline$V / \AA^{3}$ & $5652.1(15)$ & $1694.41(12)$ & $3057.4(2)$ \\
\hline$Z$ & 8 & 2 & 4 \\
\hline temperature $(\mathrm{K})$ & $130(2)$ & $130(2)$ & $130(2)$ \\
\hline radiation $(\lambda, \AA)$ & 0.71073 & 0.71073 & 0.71073 \\
\hline$\rho$ (calcd.) $\mathrm{g} \mathrm{cm}^{-3}$ & 1.238 & 1.276 & 1.264 \\
\hline$\mu(\mathrm{Mo} \mathrm{K \alpha}), \mathrm{mm}^{-1}$ & 0.407 & 0.353 & 0.384 \\
\hline$\theta$ max, deg. & 32.997 & 33.132 & 33.157 \\
\hline no. of data collected & 98275 & 30834 & 54479 \\
\hline no. of data & 10415 & 12023 & 11151 \\
\hline no. of parameters & 409 & 364 & 350 \\
\hline$R_{1}[I>2 \sigma(I)]$ & 0.0353 & 0.0286 & 0.0262 \\
\hline$w R_{2}[I>2 \sigma(I)]$ & 0.0776 & 0.0745 & 0.0664 \\
\hline$R_{1}$ [all data] & 0.0557 & 0.0326 & 0.0321 \\
\hline$w R_{2}$ [all data] & 0.0872 & 0.0762 & 0.0700 \\
\hline GOF & 1.019 & 1.078 & 1.056 \\
\hline$R_{\text {int }}$ & 0.0597 & 0.0234 & 0.0394 \\
\hline
\end{tabular}


Table S2. Crystal, Intensity, Collection, and Refinement Data for 5, 6, and 7

\begin{tabular}{|c|c|c|c|}
\hline & 5 & 6 & 7 \\
\hline lattice & Monoclinic & Monoclinic & Monoclinic \\
\hline formula & $\mathrm{C}_{30} \mathrm{H}_{47} \mathrm{NZr}$ & $\mathrm{C}_{32} \mathrm{H}_{49} \mathrm{NOZr}$ & $\mathrm{C}_{27} \mathrm{H}_{39} \mathrm{OZr}$ \\
\hline formula weight & 512.90 & 554.94 & 470.80 \\
\hline space group & $P 2_{1} / c$ & $P 2_{1} / n$ & $P 2_{1} / c$ \\
\hline$a / \AA ̊$ & $11.0833(8)$ & $12.101(3)$ & $18.0724(13)$ \\
\hline$b / \AA$ & $15.6680(12)$ & $18.466(4)$ & $9.5990(7)$ \\
\hline$c / \AA$ & $15.4308(12)$ & $13.140(3)$ & $29.022(2)$ \\
\hline$\alpha /^{\circ}$ & 90 & 90 & 90 \\
\hline$\beta /^{\circ}$ & $92.6050(10)$ & $93.447(3)$ & $94.9410(10)$ \\
\hline$\gamma /^{\circ}$ & 90 & 90 & 90 \\
\hline$V / \AA^{3}$ & $2676.8(4)$ & $2931.0(11)$ & $5015.9(6)$ \\
\hline$Z$ & 4 & 4 & 8 \\
\hline temperature $(\mathrm{K})$ & $130(2)$ & $130(2)$ & $130(2)$ \\
\hline radiation $(\lambda, \AA)$ & 0.71073 & 0.71073 & 0.71073 \\
\hline$\rho$ (calcd.) $\mathrm{g} \mathrm{cm}^{-3}$ & 1.273 & 1.258 & 1.247 \\
\hline$\mu(\mathrm{Mo} \mathrm{K} \alpha), \mathrm{mm}^{-1}$ & 0.428 & 0.399 & 0.453 \\
\hline$\theta$ max, deg. & 33.132 & 33.153 & 26.372 \\
\hline no. of data collected & 47504 & 52629 & 58869 \\
\hline no. of data & 9737 & 10660 & 10224 \\
\hline no. of parameters & 303 & 334 & 546 \\
\hline$R_{1}[I>2 \sigma(I)]$ & 0.0329 & 0.0306 & 0.0691 \\
\hline$w R_{2}[I>2 \sigma(I)]$ & 0.0777 & 0.0753 & 0.2412 \\
\hline$R_{1}$ [all data] & 0.0470 & 0.0400 & 0.0738 \\
\hline$w R_{2}$ [all data] & 0.0845 & 0.0798 & 0.2455 \\
\hline GOF & 1.039 & 1.047 & 2.405 \\
\hline$R_{\text {int }}$ & 0.0449 & 0.0325 & 0.0421 \\
\hline
\end{tabular}


Table S3. Crystal, Intensity, Collection, and Refinement Data for 1 and 8.

\begin{tabular}{|c|c|c|}
\hline & 1 & 8 \\
\hline lattice & Monoclinic & Triclinic \\
\hline formula & $\mathrm{C}_{26} \mathrm{H}_{40} \mathrm{Zr}$ & $\mathrm{C}_{28} \mathrm{H}_{40} \mathrm{O}_{2} \mathrm{Zr}$ \\
\hline formula weight & 443.80 & 499.82 \\
\hline space group & $P 2_{1} / n$ & $P-1$ \\
\hline$a / \AA ̊$ & $8.5133(4)$ & $9.4695(4)$ \\
\hline$b / \AA$ & $14.4869(6)$ & $14.8858(6)$ \\
\hline$c / \AA$ & $37.9647(17)$ & $18.8709(7)$ \\
\hline$\alpha /^{\circ}$ & 90 & $102.4550(10)$ \\
\hline$\beta /^{\circ}$ & $96.4790(10)$ & $103.7960(10)$ \\
\hline$\gamma /{ }^{\circ}$ & 90 & $97.5750(10)$ \\
\hline$V / \AA^{3}$ & $4660.8(4)$ & $2474.98(17)$ \\
\hline$Z$ & 8 & 4 \\
\hline temperature (K) & $130(2)$ & $130(2)$ \\
\hline radiation $(\lambda, \AA)$ & 0.71073 & 0.71073 \\
\hline$\rho$ (calcd.) $\mathrm{g} \mathrm{cm}^{-3}$ & 1.256 & 1.341 \\
\hline$\mu(\mathrm{Mo} \mathrm{K \alpha}), \mathrm{mm}^{-1}$ & 0.480 & 0.466 \\
\hline$\theta$ max, deg. & 30.637 & 33.105 \\
\hline no. of data collected & 75699 & 44937 \\
\hline no. of data & 14356 & 17571 \\
\hline no. of parameters & 511 & 583 \\
\hline$R_{I}[I>2 \sigma(I)]$ & 0.0427 & 0.0353 \\
\hline$w R_{2}[I>2 \sigma(I)]$ & 0.840 & 0.0884 \\
\hline$R_{I}$ [all data] & 0.0773 & 0.0416 \\
\hline$w R_{2}$ [all data] & 0.0951 & 0.0925 \\
\hline GOF & 1.012 & 1.041 \\
\hline$R_{\text {int }}$ & 0.0756 & 0.0276 \\
\hline
\end{tabular}




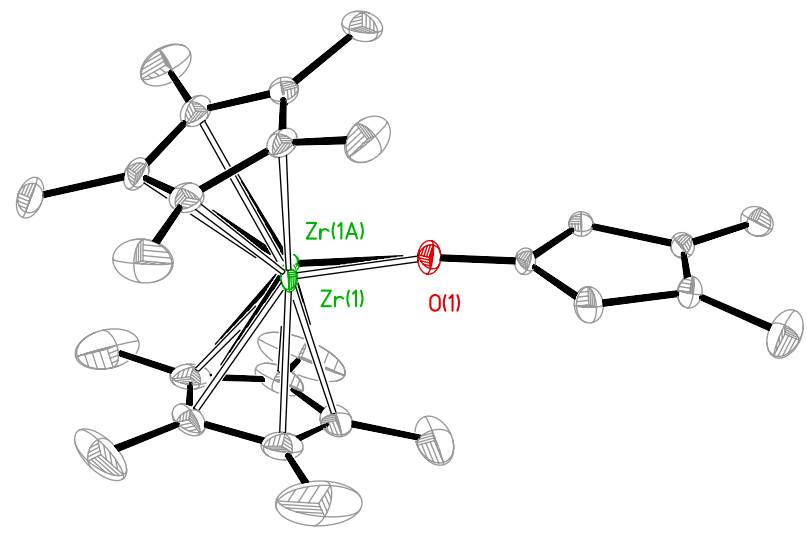

Figure S1. Molecular Structure of 7.

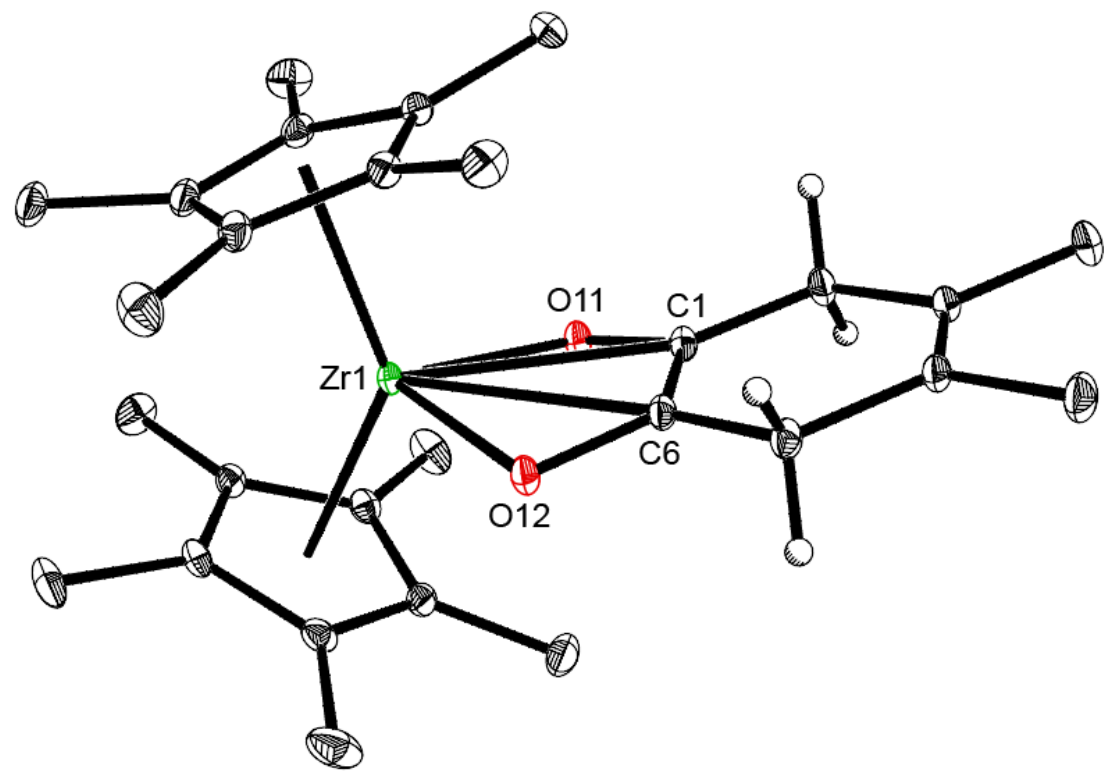

Figure S2. Molecular Structure of 8. 


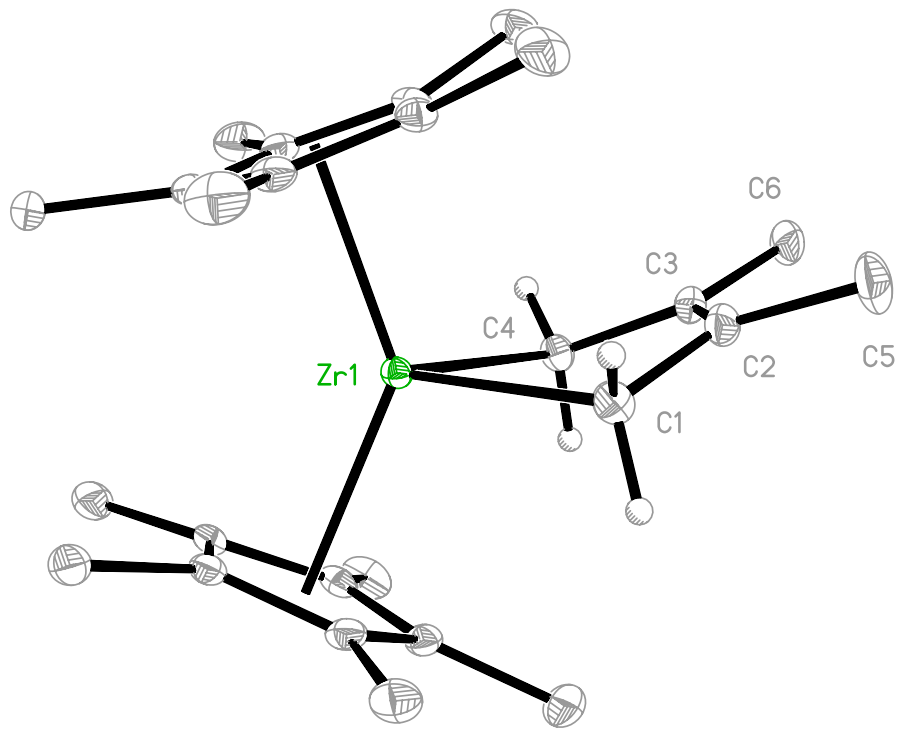

Figure S3. Molecular Structure of 1. 


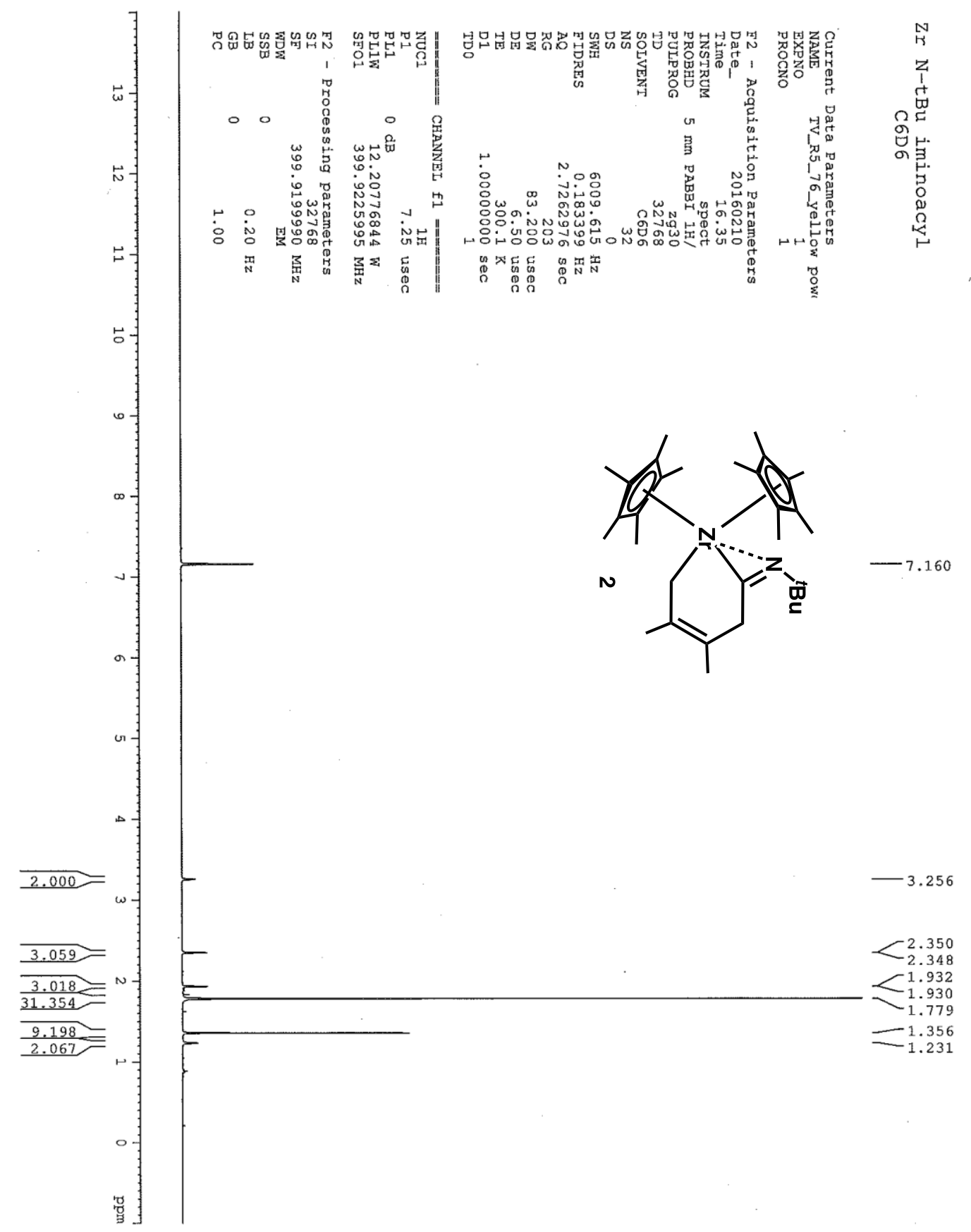




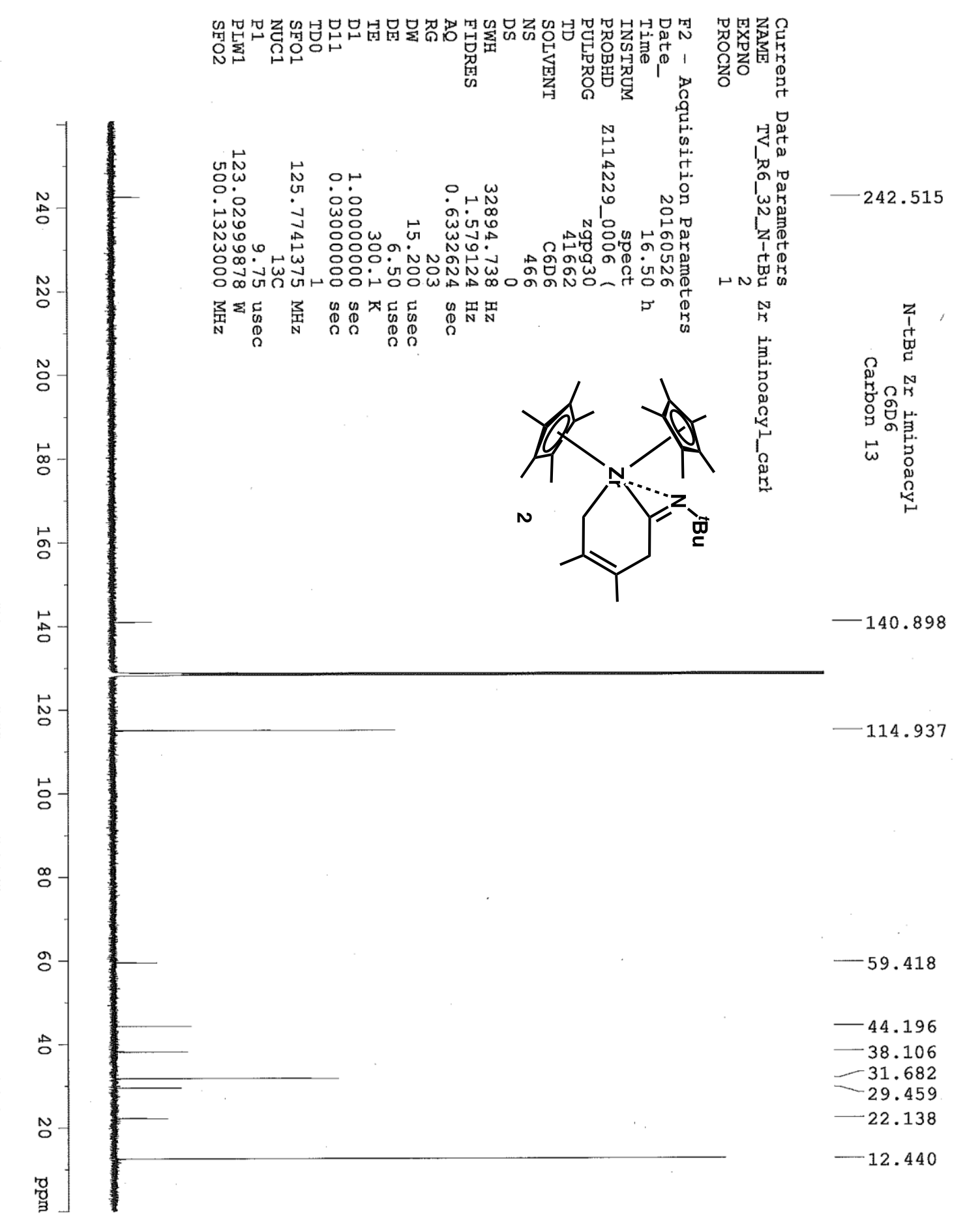




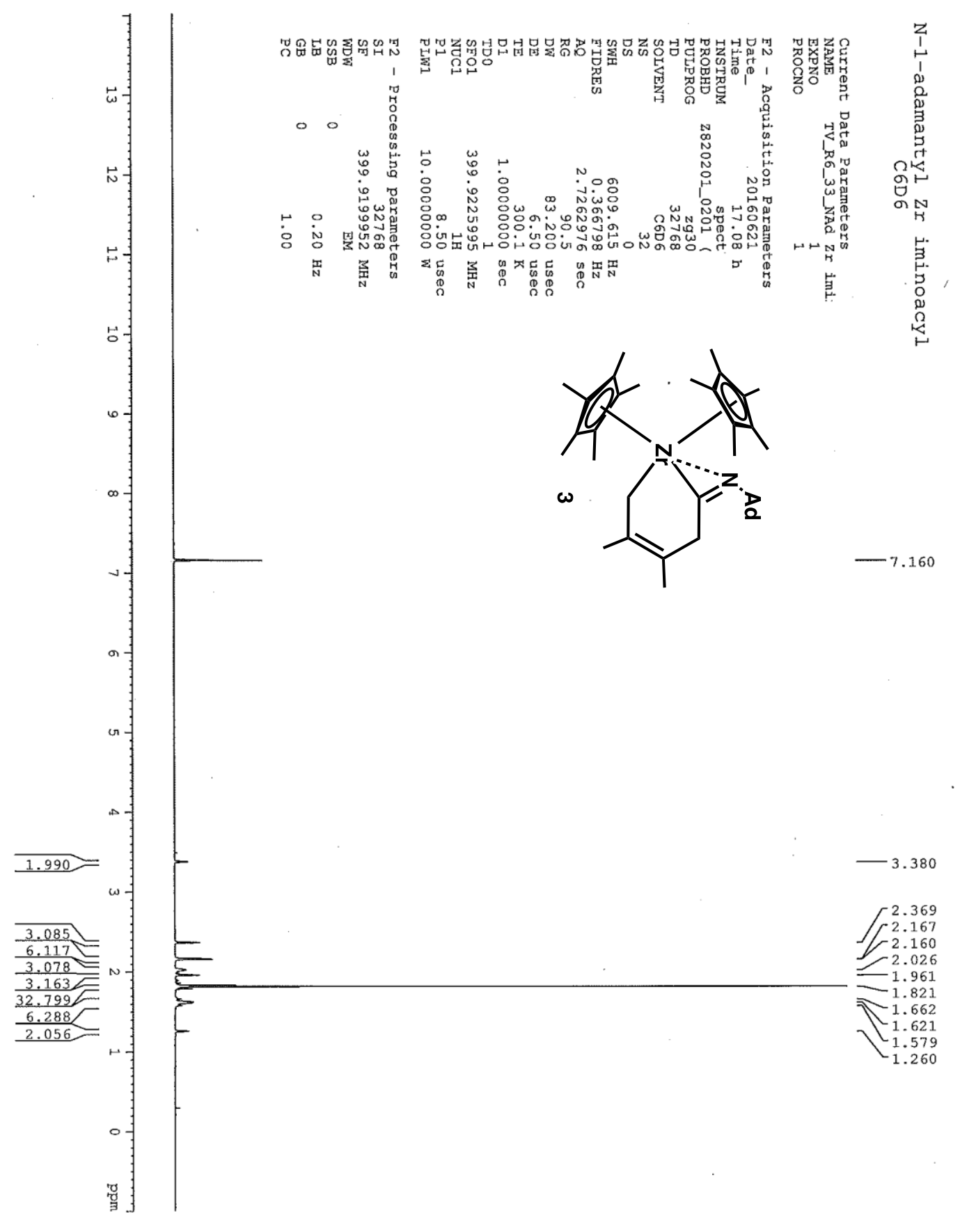




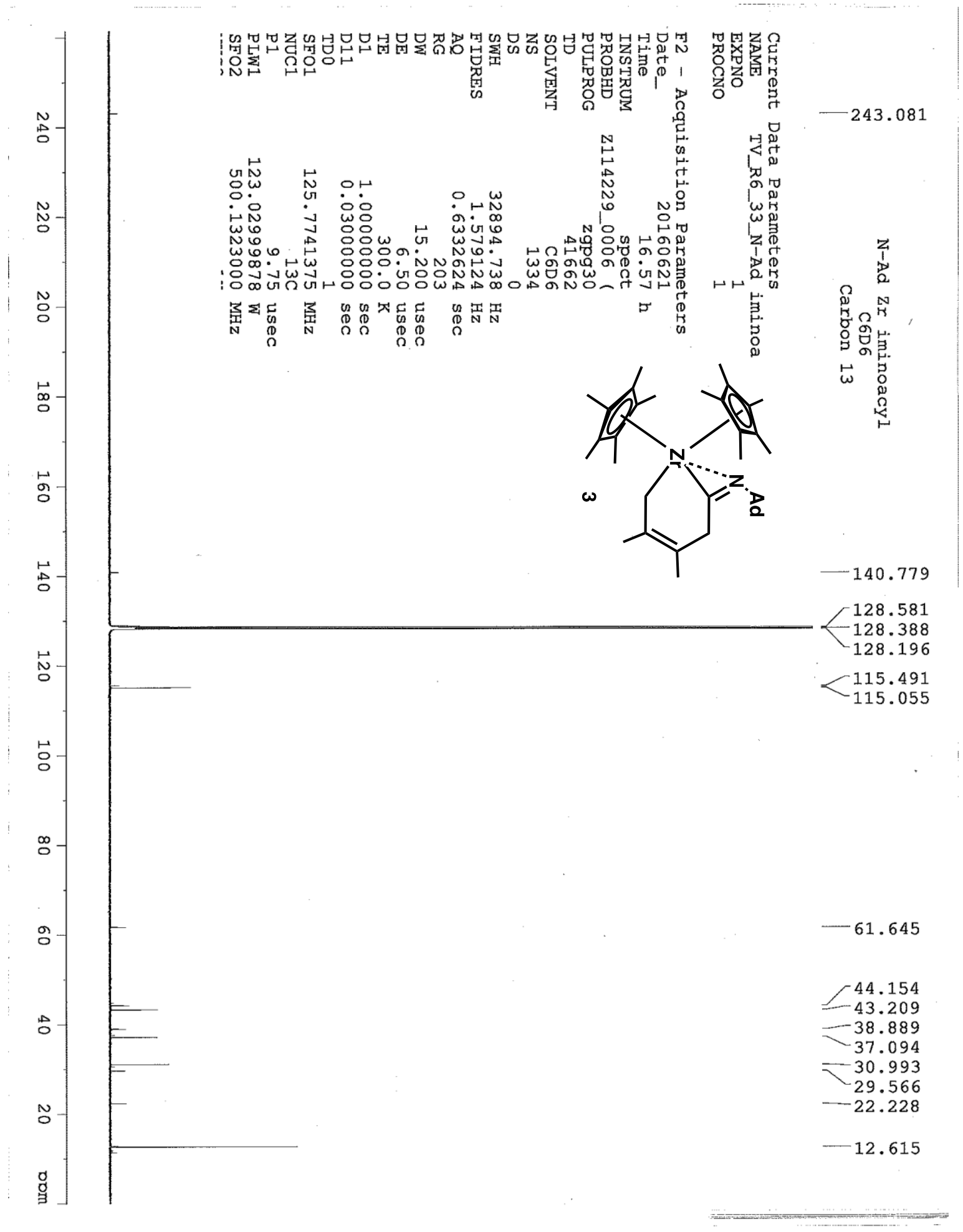




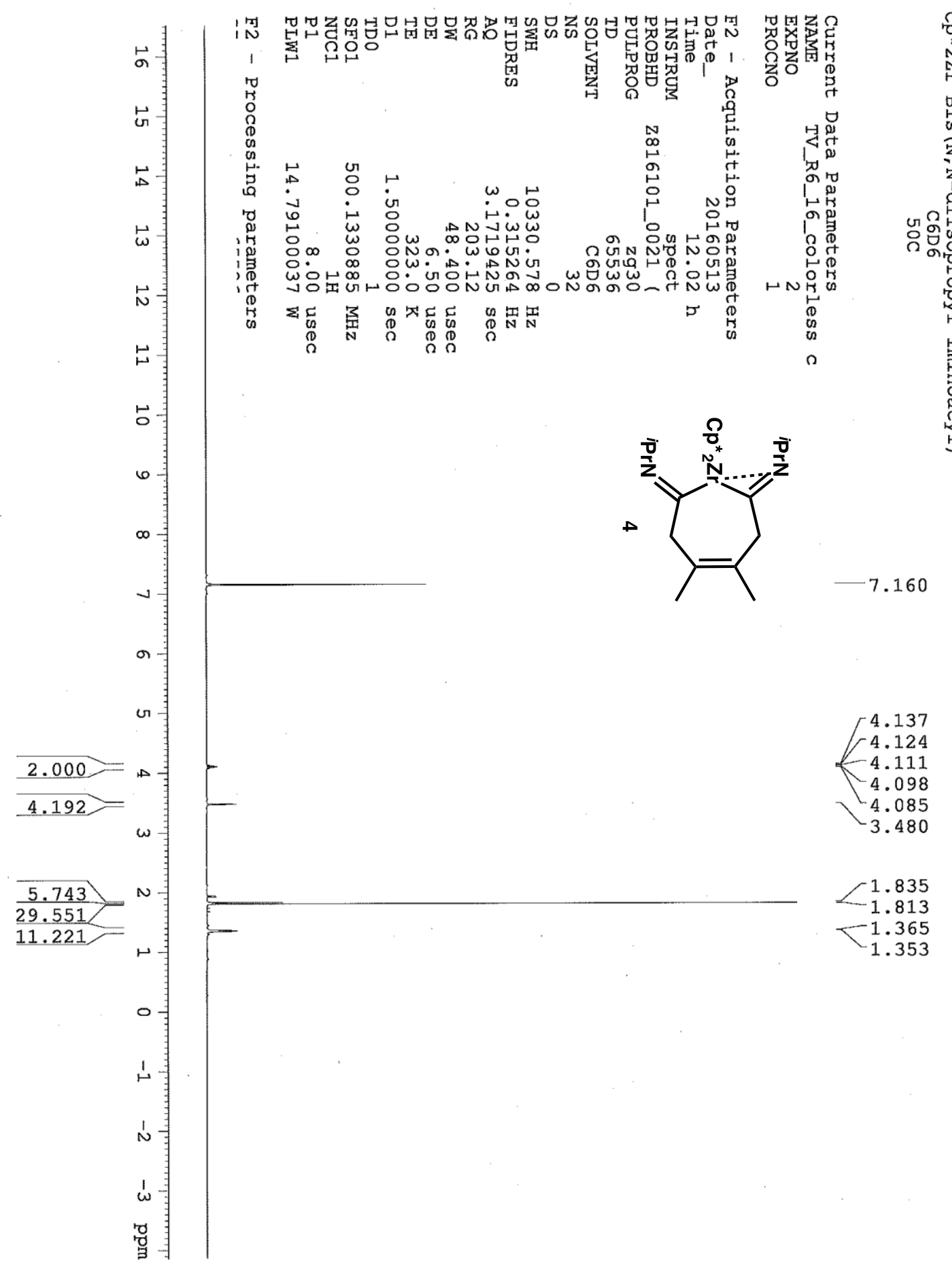




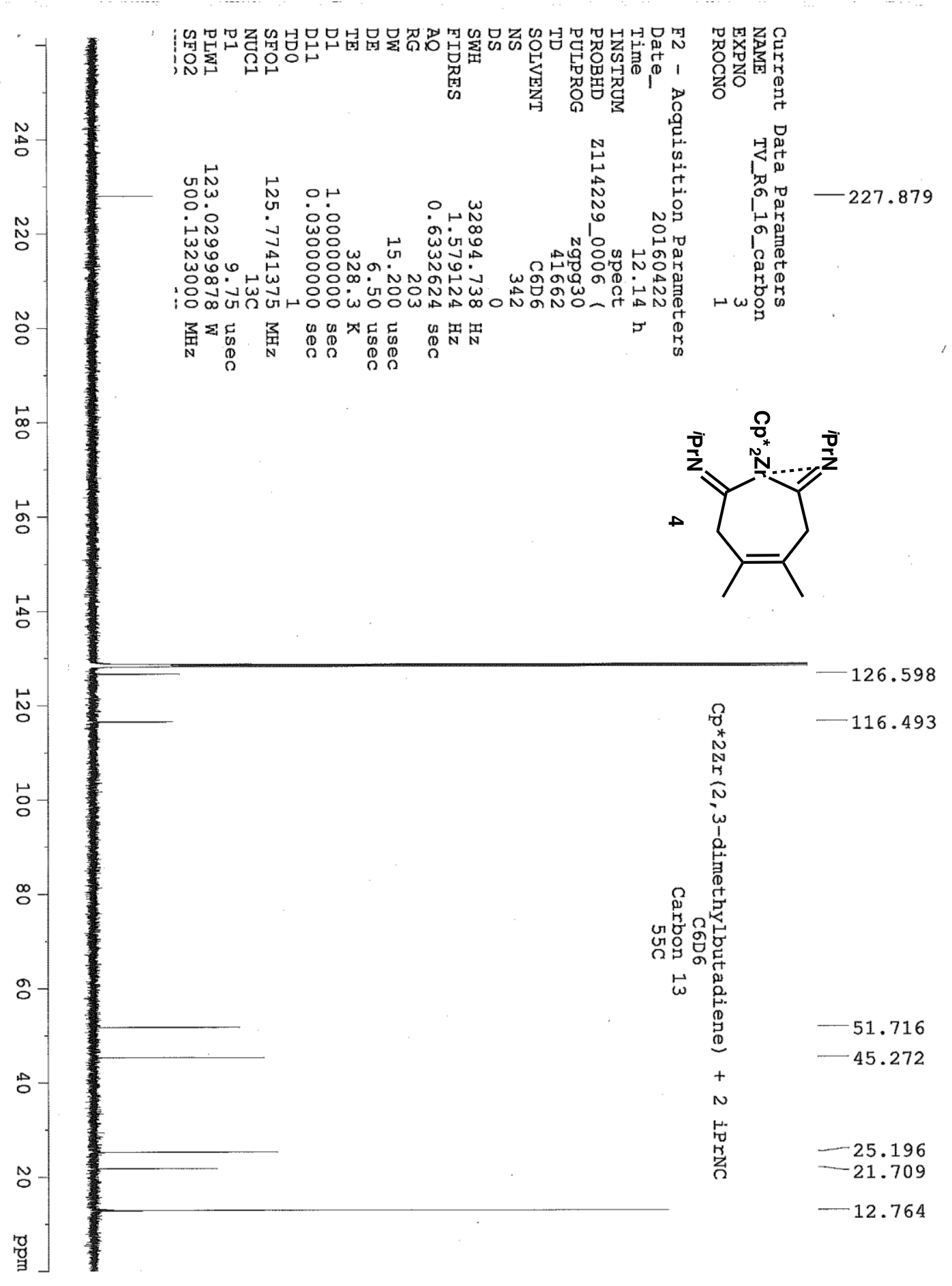




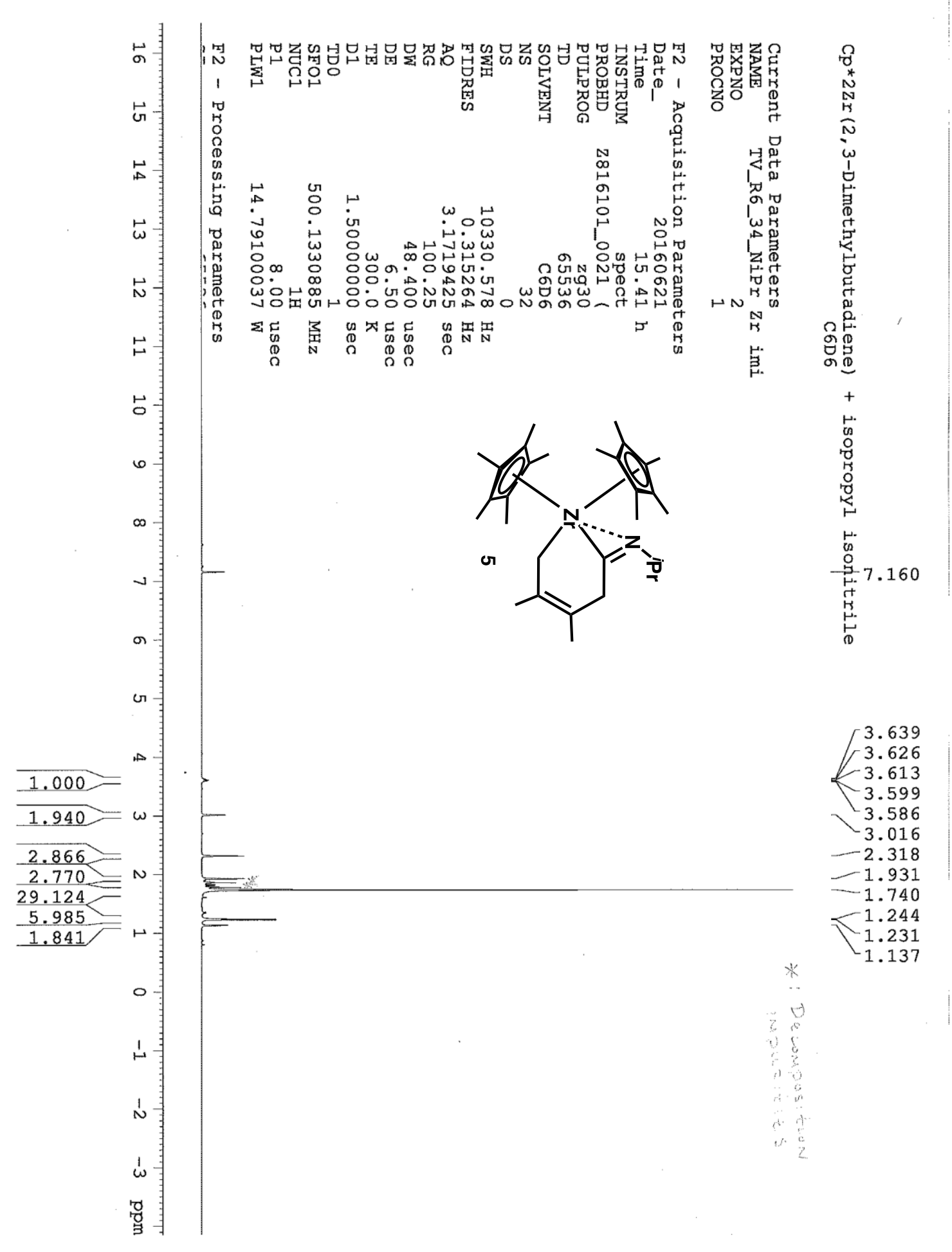




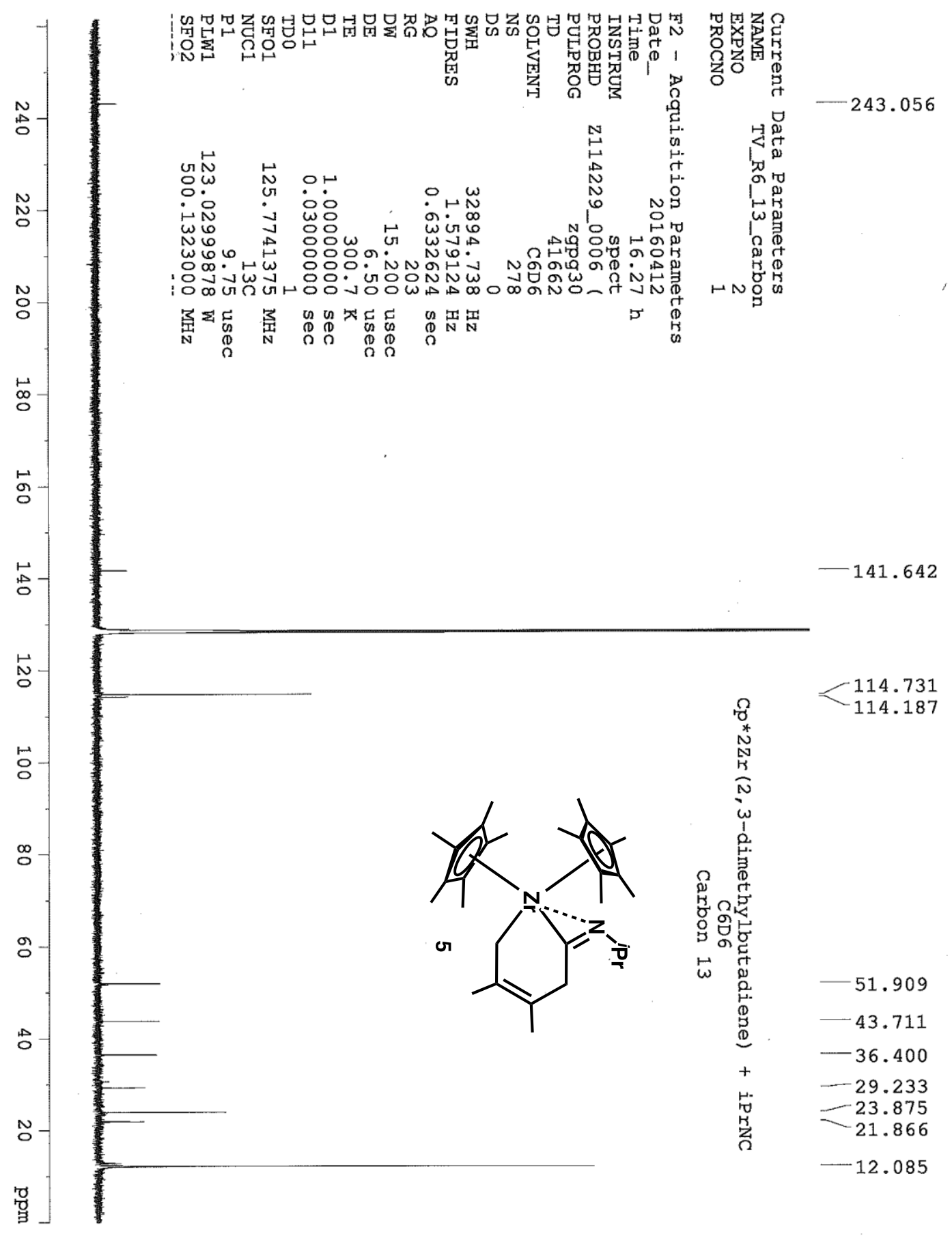




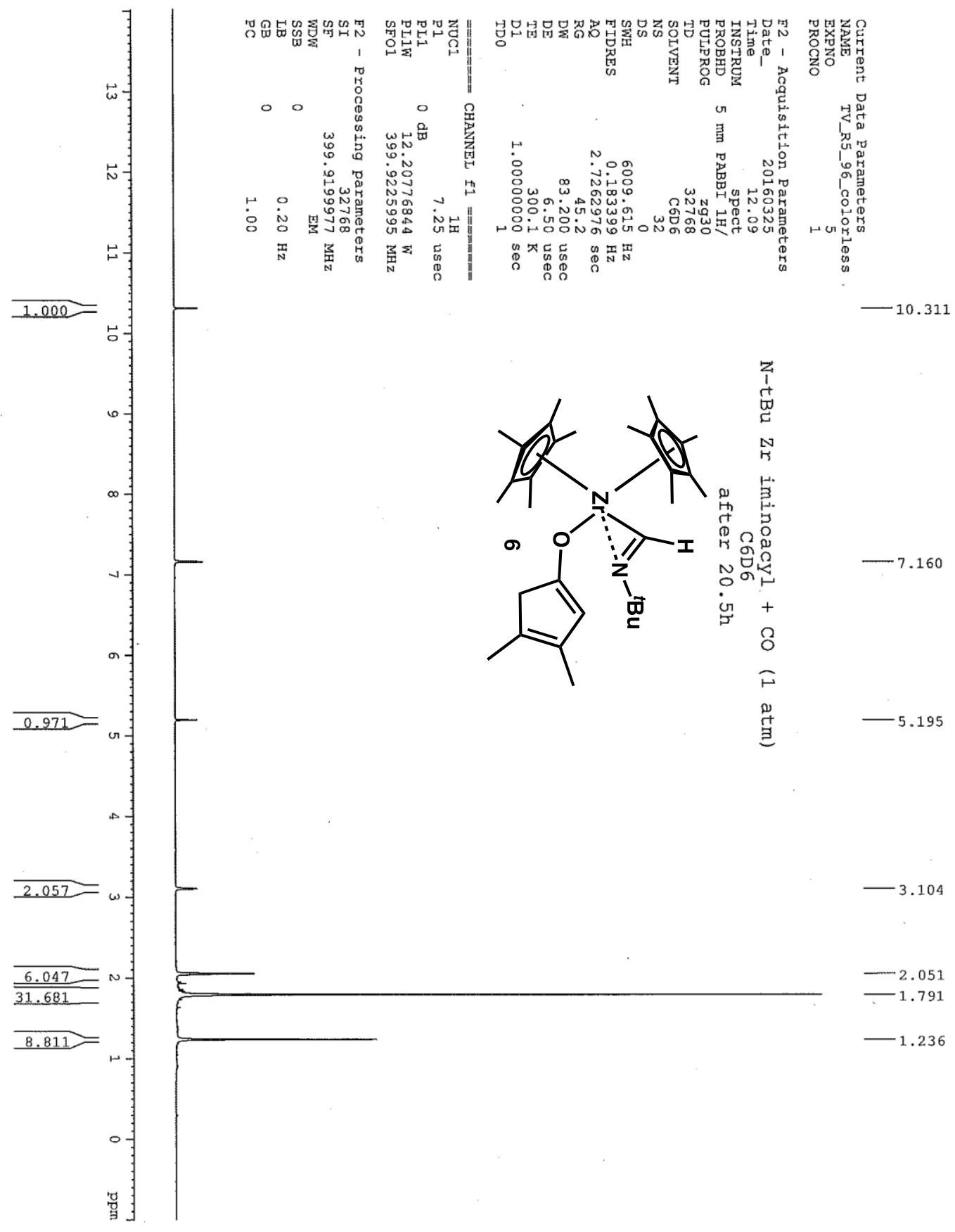




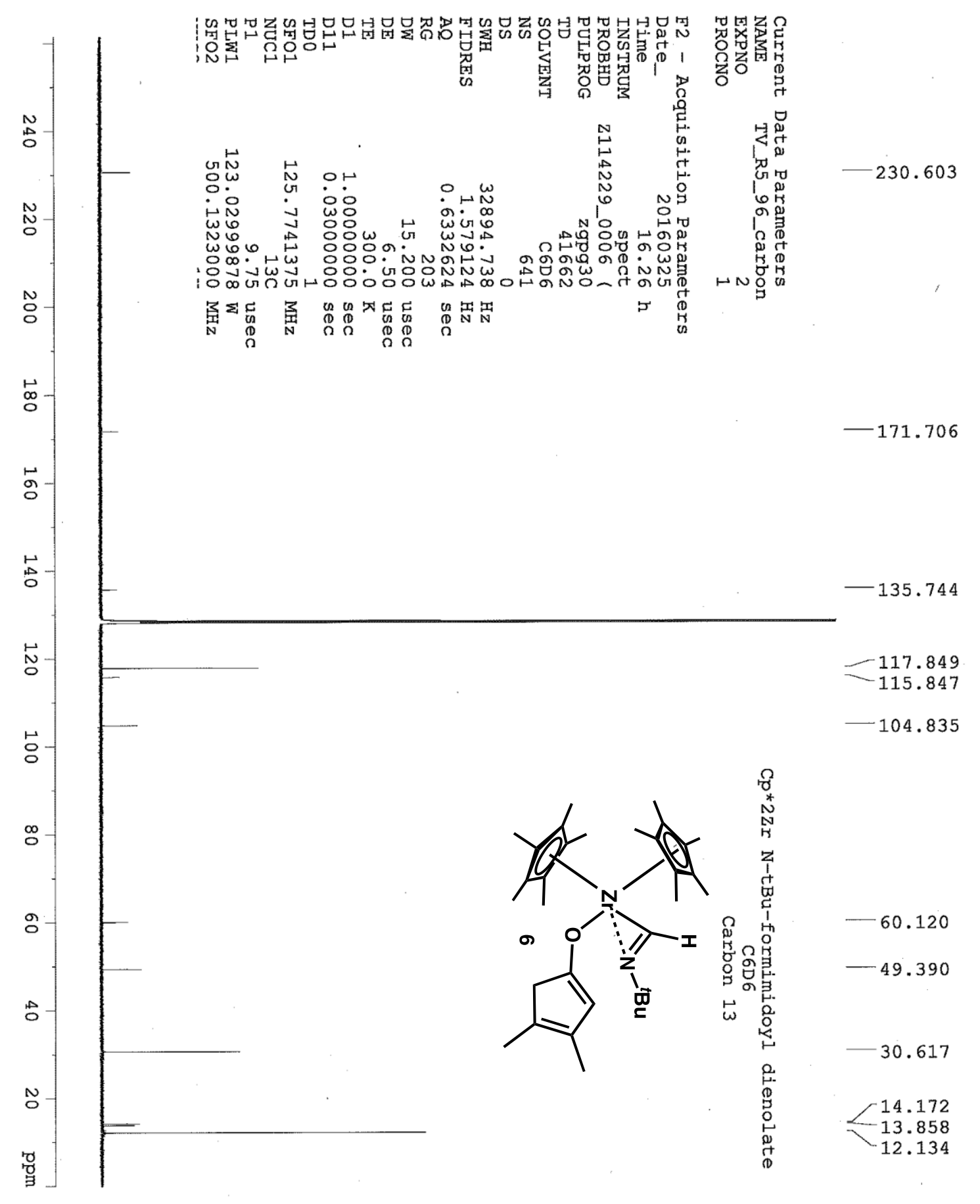




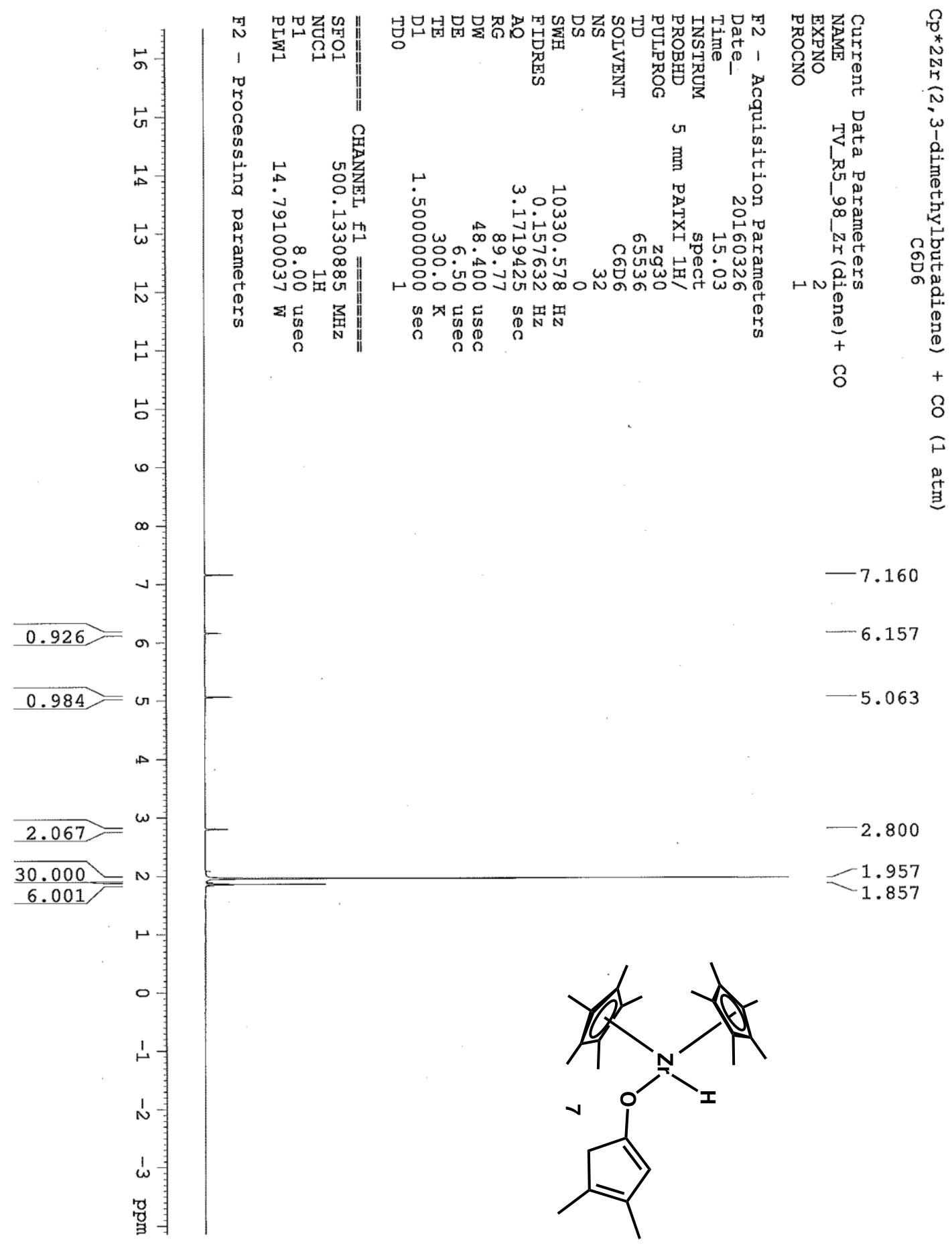




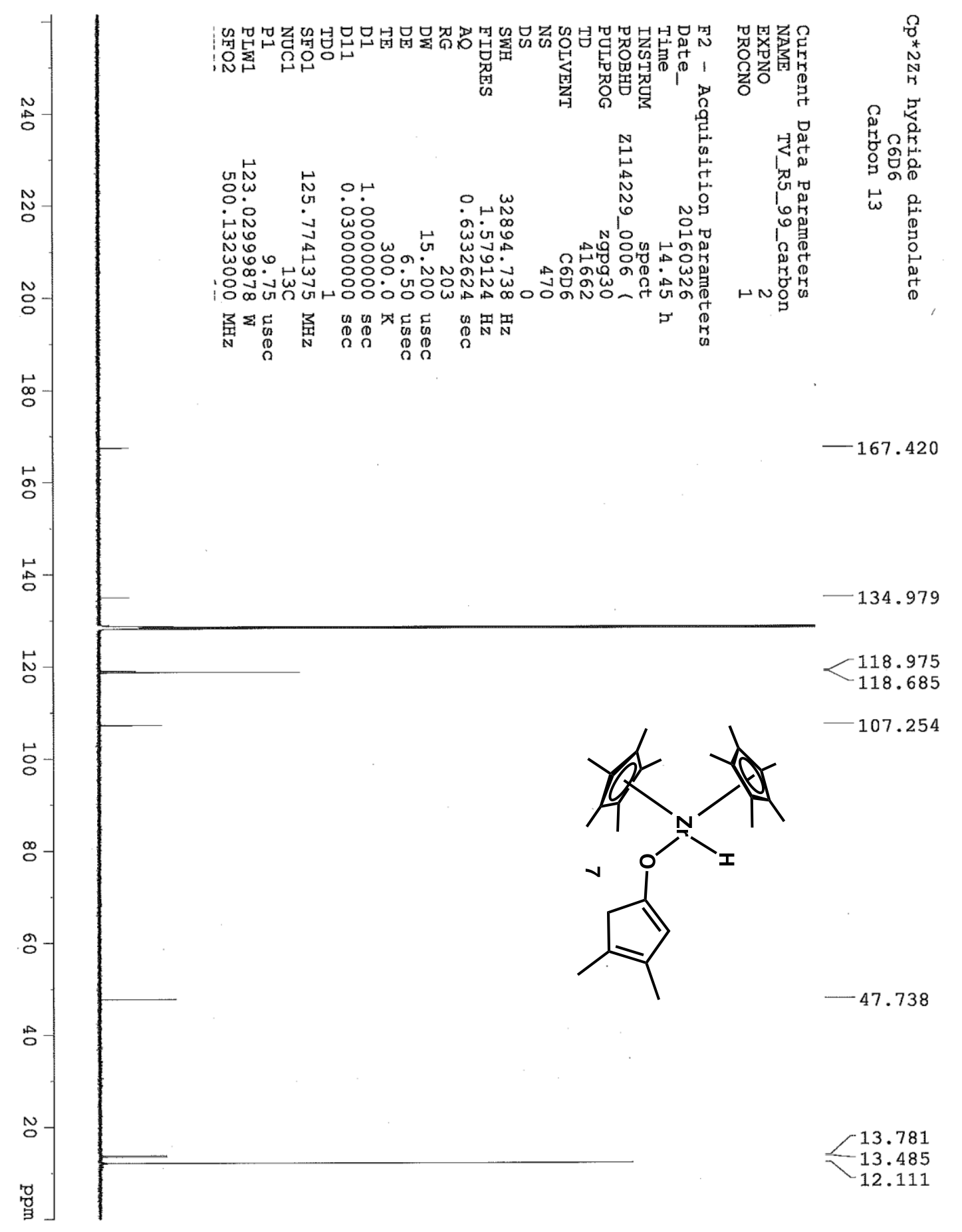

